\title{
Selective Androgen Receptor Modulator in a Patient With Hormone-Positive Metastatic Breast Cancer
}

\author{
Namratha Vontela, MD; Vamsi Koduri, MD; Lee S. Schwartzberg, MD; and Gregory A. Vidal, MD, PhD
}

\section{Abstract}

Androgen receptors (ARs) are highly coexpressed in estrogen receptor (ER)-positive breast cancers. Their role in breast tumorigenesis has been postulated, but the mechanism is not yet well-characterized. Steroidal androgens were previously used as an anticancer strategy but fell out of favor because of toxicity and the discovery of alternative therapies. Recent attempts to modulate androgen pathway signaling have focused on AR inhibitors. This report discusses a case using a well-tolerated selective AR modulator to treat a highly pretreated patient with ER-positive breast cancer, which resulted in a durable partial response.

J Natl Compr Canc Netw 2017;15(3):284-287

In estrogen receptor-positive $(\mathrm{ER}+)$ breast cancer, androgen receptor (AR) expression occurs in $67 \%$ to $88 \%$ of cases and is associated with an improved outcome. ${ }^{1}$ Before development of selective ER modulators (SERMs), breast cancer was treated with testosterone and its derivatives with some clinical efficacy. ${ }^{2}$ However, androgen therapy fell out of favor due to concerns of aromatization to its virilizing effects, and the availability of tamoxifen and third-generation aromatase inhibitors (AIs). ${ }^{3}$ Resistance to antiestrogen therapy nevertheless develops in most patients with ER+ advanced breast cancer, and new treatment approaches are needed. Antiandrogens such as bicalutamide and enzalutamide have shown promise in patients with AR-positive $(\mathrm{AR}+)$ breast cancer. ${ }^{4,5}$ Targeting the AR through the development of selective AR modulators (SARMs), with the potential for a reduced toxicity profile, represents a new opportunity for endocrine therapy. GTx024 (enobosarm) (GTx, Inc., Memphis, TN) is a novel oral nonsteroidal SARM with diminished androgenic effects, which binds to the AR with similar affinity to testosterone and has inhibitory effects on breast cancer cell growth in vitro and in vivo. Enobosarm is currently being tested in phase II trials in both $\mathrm{AR}+, \mathrm{ER}+$, and

From the University of Tennessee West Cancer Center, Germantown, Tennessee.

Submitted August 25, 2016; accepted for publication December 20, 2016.

Dr. Schwartzberg has disclosed that he serves on the advisory board

for GTx, Inc. The remaining authors have disclosed that they have no
$\mathrm{AR}+$ triple-negative subtypes of breast cancer. ${ }^{6}$ This report presents a case of a heavily pretreated woman with metastatic ER+/progesterone receptor-positive $(\mathrm{PR}+)$ / HER2 - breast cancer with high AR coexpression who achieved partial radiographic response after 4 cycles of treatment with enobosarm in a clinical trial.

\section{Case Presentation}

A 65-year-old white woman presented to our clinic who was initially diagnosed with clinical stage IIB (T2N1M0) ER+/PR+, HER2- infiltrating ductal carcinoma of the breast in 2007. She received neoadjuvant chemotherapy with dose-dense doxorubicin, cyclophosphamide, and paclitaxel, and subsequently underwent a lumpectomy and axillary lymph node dissection showing residual carcinoma. After whole-breast radiation, she was maintained on adjuvant anastrozole (AI) until February 2013, when a PET/CT demonstrated metastatic bone-only disease; a biopsy confirmed metastatic breast cancer $(\mathrm{mBC})$. Her therapy was then switched to fulvestrant and denosumab on April 4, 2013, until a surveillance CT scan in December 2014 showed a new liver lesion. Biopsy of the liver lesion confirmed 90\%

financial interests, arrangements, affiliations, or commercial interests with the manufacturers of any products discussed in this article or their competitors.

Correspondence: Gregory A. Vidal, MD, PhD, University of Tennessee West Cancer Center, 7945 Wolf River Boulevard, Germantown, TN 38139.

E-mail: gvidal@westclinic.com 
$\mathrm{ER}+, 100 \% \mathrm{PR}+$, and HER2- (1+ immunohistochemistry) $\mathrm{mBC}$. She was then switched to everolimus and exemestane, but only tolerated 2 weeks of therapy due to mucositis and the development of a macular skin rash.

On February 25, 2015, she was enrolled on an expanded access program of palbociclib, $125 \mathrm{mg} / \mathrm{d}$ and letrozole, $2.5 \mathrm{mg}$. Additionally, a comprehensive molecular profile was performed on the liver biopsy specimen. Pertinent immunohistochemistry results confirmed ER and PR positivity (95\% and 90\%, respectively) and revealed AR positivity (95\%). Nextgeneration testing also revealed KRAS G12D and TP53 N4239S mutations. Following cycle 2 of palbociclib and letrozole, CT scans showed stable disease. Repeat imaging performed on August 27, 2015, after 6 cycles of treatment showed disease progression, with PET/CT demonstrating multiple, new, low-density lesions scattered throughout both lobes of the liver and evidence of progressive bony metastatic disease in the right and left iliac crests and acetabulum. Because of complaints of new headaches, a brain MRI with contrast was performed, which showed an enhancing choroid plexus with no intracranial metastasis, but with calvarial metastases; the patient was otherwise asymptomatic. She subsequently met criteria for the phase II single-arm study evaluating the efficacy and safety of enobosarm in $\mathrm{AR}+, \mathrm{ER}+, \mathrm{HER} 2-\mathrm{mBC}$ (ClinicalTrials.gov identifier: NCT02463032).

After meeting the screening requirement, she was enrolled on enobosarm at $18 \mathrm{mg} / \mathrm{d}$ orally, given on a 28-day cycle schedule. At initiation of enobosarm, on September 25, 2015, a CT of the chest, abdomen, and pelvis (CT-CAP) with contrast was performed; results showed disease progression, with disease in the bone and liver lesions increasing by approximately $20 \%$. Baseline measurements of the measurable liver lesions 1 and 2 were $20 \times 22 \mathrm{~mm}$ and $22 \times 23 \mathrm{~mm}$, respectively (Figure 1). After cycle 3 of treatment, surveillance scans on December 24, 2015 , documented a size reduction to $19 \times 16 \mathrm{~mm}$ and $22 \times 24 \mathrm{~mm}$, respectively (Figure 1). After cycle 6 , a confirmation scan (March 11, 2016) reported a further $43 \%$ reduction from baseline for lesions 1 and $2(10 \times 9 \mathrm{~mm}$ and $14 \times 9 \mathrm{~mm}$, respectively; Figure 1). Repeat CT-CAP on June 16, 2016, revealed stable disease per RECIST criteria with a slight progression of lesion $1(15 \times 12 \mathrm{~mm})$ but with further reduction in lesion 2 (9 $\times 10 \mathrm{~mm})$ compared with her previous

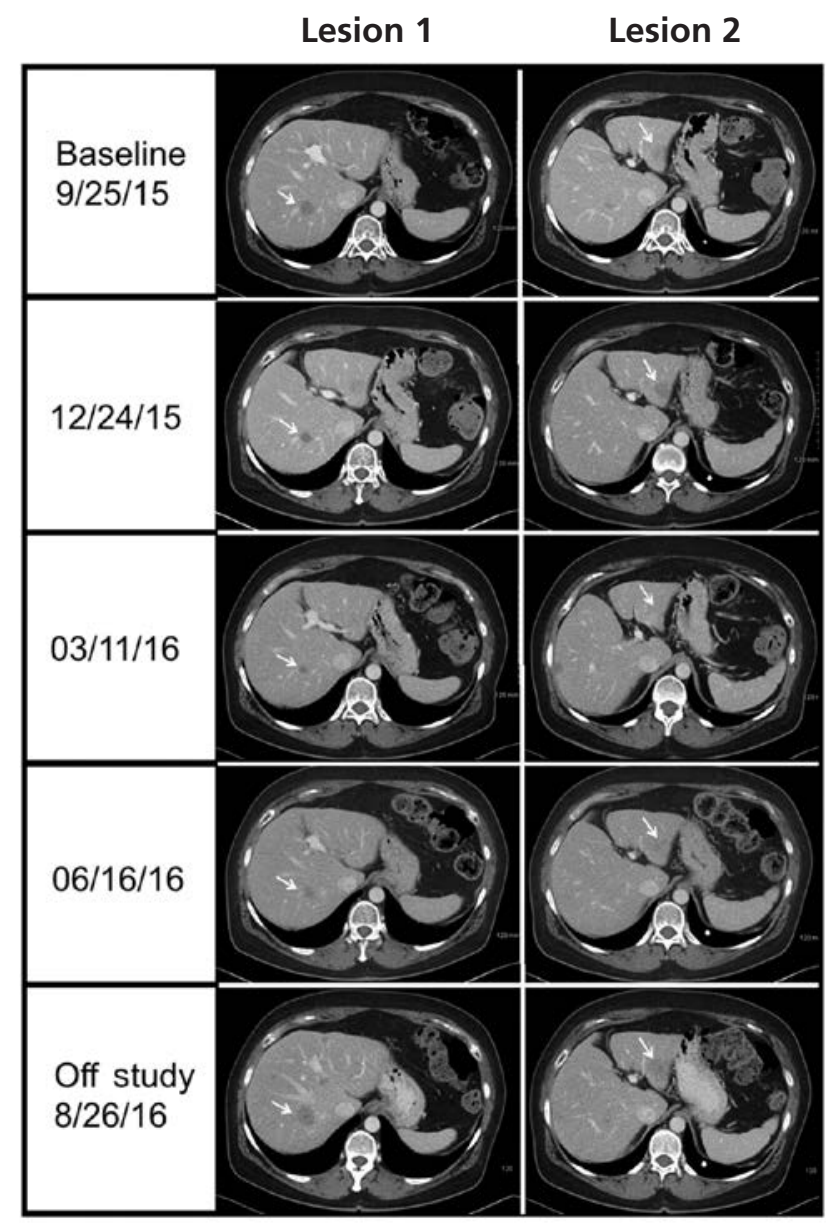

Figure 1. Comparison of target lesions (white arrows).

scan. Unfortunately, a CT-CAP performed on August 26, 2016, revealed disease progression; several new, small hepatic lesions; and growth of both liver lesions to $32 \times 25 \mathrm{~mm}$ and $30 \times 24$, respectively, reflecting an 11-month progression-free survival.

Bone scan lesions responded to therapy as well. A baseline bone scan performed on September 25, 2015, was read as probable metastasis in right calvarium, left fourth rib posteriorly, right pelvis, and spinous process at the cervicothoracic joint (image not shown). Interval bone scan performed on December 17, 2015, documented persistent activity in the calvarium, rib, pelvis, spinous process, and cervicothoracic joint. A repeat bone scan on April 15, 2016, demonstrated stable disease with no new abnormalities.

Treatment-emergent adverse events included grade 1 alopecia, facial acne, and fatigue; all adverse events were attributed to the trial medications. Her facial acne resolved mostly with topical mupirocin and 
Vontela et al

Cleocin antibiotic ointment. The patient remained on trial for 10 cycles and then was rechallenged with exemestane and everolimus (dose-reduced).

\section{Discussion}

$\mathrm{ER}+\mathrm{mBC}$ remains an incurable disease. Guidelines from NCCN and ASCO currently recommend treating patients through sequential lines of endocrine therapy until progressive disease resistance occurs, at which time chemotherapy is indicated. ${ }^{7,8}$ Multiple classes of agents targeting ERs are useful, and include SERMs, AIs, and selective ER degraders (SERDs). As observed in our patient, ER expression is maintained in most tumors that are initially $\mathrm{ER}+$, but resistance subsequently develops over time ${ }^{9}$; this suggests that genetic or epigenetic changes within the tumor may activate hormoneindependent mitogenic pathways leading to antiestrogen resistance. ${ }^{10}$ Newer agents such as everolimus and palbociclib, which mitigate alternative signaling pathways, including the mTOR/PI3K and cell cycle, improve progression-free survival, but eventually endocrine resistance does occur. ${ }^{6}$ Mutations in the ER-alpha (ERa) gene ESR1 have been identified that appear to confer resistance to AIs but not SERDs. ${ }^{11,12}$ Identifying additional mechanistic drivers of resistance and defining ways to overcome them is of utmost importance.

Our patient had been exposed to, and benefited from, a number of endocrine therapies, but ultimately experienced disease progression through each, and required alternative therapeutic interventions. Chemotherapy remained an option, but given her excellent performance status, relatively low volume of disease, and desire to postpone chemotherapy as an absolute last resort, we sought other therapeutic targets.

ARs have emerged recently as a target in breast cancer. As in our patient, the AR is highly coexpressed with the ER in ER+ breast cancers, and may play a role in breast tumorigenesis. ${ }^{13}$ Interestingly, AR coexpression in ER+ breast cancer appears to be a positive prognostic factor. ${ }^{14-17}$ For example, ER+ tumors with AR positivity $>75 \%$ have been shown to be associated with a significantly improved overall survival compared with those with low AR expression. ${ }^{14}$ Furthermore, tumors expressing high levels of AR were noted to have a significant 5-year overall survival benefit compared with low expressers of $A R$, in- dependent of variables including ER, PR, HER2; PIK3CA mutational status; and prior treatments. ${ }^{16}$

The exact mechanism of action of the AR in breast cancer is not yet well understood. It has been suggested that the AR may modulate ER signaling through ER-beta (ER $\beta)$, which augments ER $\alpha$ nuclear translocation, thus suppressing metastasis, and leading to improved outcomes. ${ }^{18-20}$ AR activation may increase $\operatorname{ER} \beta$ promoter activity while inhibiting ER $\alpha$ activity. ${ }^{14,21}$ In contrast, AR overexpression has also been shown to inhibit ER $\alpha$ and confer resistance to tamoxifen. ${ }^{22}$ This contradistinction may be dependent on the dominant signaling pathway.

It is postulated that tumorigenesis in $\mathrm{ER}+1$ $\mathrm{AR}+$ tumors is driven by $\geq 2$ separate processes: an estrogen-responsive and an androgen-responsive process. In the former, AR nuclear localization is an estrogen-dependent activity. ${ }^{23}$ In response to estrogen stimulation, AR nuclear localization favors genomic binding at sites enriched for estrogen-responsive elements, resulting in activation of cellular proliferative pathways. Conversely, in the androgenresponsive process, activation of the $A R$ and the consequential nuclear localization favors activation of estrogen-independent cellular survival pathways. Inhibitory effects of the antiandrogen enzalutamide appears independent of the dominant process. ${ }^{23}$ Two independent phase II clinical studies demonstrated that the inhibition of the AR with antiandrogens, bicalutamide or enzalutamide had clinical activity in ER-/PR- breast cancers. ${ }^{5,24}$

Enobosarm is a novel, oral nonsteroidal SARM that does not undergo aromatization when bound to the aromatase enzyme nor is it a substrate of $5 \alpha$-reductase. ${ }^{25}$ It may also harbor tissue specific activity, though the exact mechanism is unknown. ${ }^{26}$ Therefore, it can be hypothesized that in postmenopausal women with ER+ breast cancer the binding of a nonsteroidal SARM to the aromatase enzyme could result in reduced estrogen production given its inability to be aromatized, thus directly competing with androgens for the aromatase-binding site. Alternatively, nonsteroidal SARMS also may activate differential coregulators and transcription factors of the androgen pathway, leading to antitumor activity. ${ }^{25,26}$

Enobosarm has been extensively evaluated in multiple studies enrolling $>1,500$ patients for catabolic effects in anorexia. ${ }^{27} \mathrm{It}$ is tolerated in doses up to $30 \mathrm{mg}$ per oral solution daily. In a small phase II trial 
of enobosarm evaluating 22 postmenopausal women with $\mathrm{ER}+\mathrm{mBC}$ that had previously responded to adjuvant hormonal therapy, the primary end point was able to be assessed in $17 \mathrm{AR}+$ patients, with $35 \%$ (6/17) demonstrating a clinical benefit at 6 months. The drug was well-tolerated and was associated with improvements in bone health and libido. Additionally, it has been associated with an increase in total lean muscle mass, improvement in lean muscle mass, and a decrease in insulin resistance in a small phase II trial. ${ }^{28}$ However, 2 registration placebo-controlled phase III trials failed to meet primary end points of improvement in lean body mass and physical activity at 3 months in patients with stage III and IV nonsmall cell lung cancer. ${ }^{29,30}$

\section{Conclusions}

Our patient with $\mathrm{ER}+/ \mathrm{PR}+/ \mathrm{AR}+\mathrm{mBC}$, whose disease progressed through anastrazole and fulvestrant, was intolerant of everolimus/exemestane, and then subsequently progressed through letrozole/palbociclib, is the first reported case of a clinical response to a nonsteroidal SARM after failure of multiple modern ER-targeted therapies. She continued to experience clinical benefit for 11 months before meeting criteria for progression based on RECIST criteria. Ongoing clinical investigations will be required to determine the full benefit of this approach in endocrine-resistant, $\mathrm{ER}+\mathrm{mBC}$.

\section{References}

1. Loibl S, Müller BM, von Minckwitz G, et al. Androgen receptor expression in primary breast cancer and its predictive and prognostic value in patients treated with neoadjuvant chemotherapy. Breast Cancer Res Treat 2011;130:477-487.

2. McNamara KM, Moore NL, Hickey TE, et al. Complexities of androgen receptor signalling in breast cancer. Endocr Relat Cancer 2014;21:T16181.

3. Kennedy BJ. Fluoxymesterone therapy in advanced breast cancer. N Engl J Med 1958;259:673-675.

4. Gucalp A, Tolaney S, Isakoff SJ, et al. Phase II trial of bicalutamide in patients with androgen receptor-positive, estrogen receptor-negative metastatic breast cancer. Clin Cancer Res 2013;19:5505-5512.

5. Traina TA, Miller K, Yardley DA, et al. Results from a phase 2 study of enzalutamide (ENZA), an androgen receptor (AR) inhibitor, in advanced $\mathrm{AR}+$ triple-negative breast cancer (TNBC) [abstract]. J Clin Oncol 2015;33(Suppl):Abstract 1003.

6. Clarke R, Leonessa F, Welch JN, Skaar TC. Cellular and molecular pharmacology of antiestrogen action and resistance. Pharmacol Rev 2001;53:25-72.

7. Gradishar WJ, Anderson BO, Balassanian R, et al. Invasive Breast Cancer Version, 1.2016, NCCN Clinical Practice Guidelines in Oncology. J Natl Compr Canc Netw 2016;14:324-354.

8. Rugo HS, Rumble RB, Macrae E, et al. Endocrine therapy for hormone receptor-positive metastatic breast cancer: American Society of Clinical Oncology guideline. J Clin Oncol 2016;34:3069-3103.

9. Kuukasjärvi T, Kononen J, Helin H, et al. Loss of estrogen receptor in recurrent breast cancer is associated with poor response to endocrine therapy. J Clin Oncol 1996;14:2584-2589.

10. Shou J, Massarweh S, Osborne CK, et al. Mechanisms of tamoxifen resistance: increased estrogen receptor-HER2/neu cross-talk in ER/HER2positive breast cancer. J Natl Cancer Inst 2004;96:926-935.

11. Robinson DR, Wu YM, Vats $P$, et al. Activating ESR 1 mutations in hormone-resistant metastatic breast cancer. Nat Genet 2013;45:14461451 .

12. Fribbens C, O'Leary B, Kilburn L, et al. Plasma ESR1 mutations and the treatment of estrogen receptor-positive advanced breast cancer. J Clin Oncol 2016;34:2961-2968.

13. Loibl S, Müller BM, Minckwitz von G, et al. Androgen receptor expression in primary breast cancer and its predictive and prognostic value in patients treated with neoadjuvant chemotherapy. Breast Cancer Res Treat 2011;130:477-487.

14. Peters AA, Buchanan G, Ricciardelli C, et al. Androgen receptor inhibits estrogen receptor-alpha activity and is prognostic in breast cancer. Cancer Res 2009;69:6131-6140.

15. Peters KM, Edwards SL, Nair SS, et al. Androgen receptor expression predicts breast cancer survival: the role of genetic and epigenetic events. BMC Cancer 2012;12:132.

16. Santagata S, Thakkar A, Ergonul A, et al. Taxonomy of breast cancer based on normal cell phenotype predicts outcome. J Clin Invest 2014;124:859870.

17. Garay JP, Park BH. Androgen receptor as a targeted therapy for breast cancer. Am J Cancer Res 2012;2:434-445.

18. Karamouzis MV, Papavassiliou KA, Adamopoulos C, Papavassiliou AG. Targeting androgen/estrogen receptors crosstalk in cancer. Trends Cancer 2016;2:35-48.

19. Huang B, Warner M, Gustafsson JA. Estrogen receptors in breast carcinogenesis and endocrine therapy. Mol Cell Endocrinol 2015;418(Pt 3):240-244.

20. Hartman J, Lindberg K, Morani A, et al. Estrogen receptor beta inhibits angiogenesis and growth of T47D breast cancer xenografts. Cancer Res 2006;66:11207-11213.

21. Rizza P, Barone I, Zito D, et al. Estrogen receptor beta as a novel target of androgen receptor action in breast cancer cell lines. Breast Cancer Res 2014;16:R21.

22. De Amicis F, Thirugnansampanthan J, Cui Y, et al. Androgen receptor overexpression induces tamoxifen resistance in human breast cancer cells. Breast Cancer Res Treat 2009;121:1-11.

23. D'Amato NC, Gordon MA, Babbs B, et al. Cooperative dynamics of AR and ER activity in breast cancer. Mol Cancer Res 2016;14:1054-1067.

24. Gucalp A, Tolaney S, Isakoff SJ, et al. Phase II trial of bicalutamide in patients with androgen receptor-positive, estrogen receptor-negative metastatic breast cancer. Clin Cancer Res 2013;19:5505-5512.

25. Bhasin S, Jasuja R. Selective androgen receptor modulators as function promoting therapies. Curr Opin Clin Nutr Metab Care 2009;12:232-240.

26. Narayanan R, Coss CC, Yepuru M, et al. Steroidal androgens and nonsteroidal, tissue-selective androgen receptor modulator, S-22, regulate androgen receptor function through distinct genomic and nongenomic signaling pathways. Mol Endocrinol 2008;22:2448-2465.

27. Srinath R, Dobs A. Enobosarm (GTx-024, S-22): a potential treatment for cachexia. Future Oncol 2014;10:187-194.

28. Dalton JT, Barnette KG, Bohl CE, et al. The selective androgen receptor modulator GTx-024 (enobosarm) improves lean body mass and physical function in healthy elderly men and postmenopausal women: results of a double-blind, placebo-controlled phase II trial. J Cachexia Sarcopenia Muscle 2011;2:153-161.

29. GTx reports results for enobosarm POWER trials for the prevention and treatment of muscle wasting in patients with non-small cell lung cancer. Available at: http://www.businesswire.com/news/home/20130819005378/ en/GTx-Reports-Results-Enobosarm-POWER-Trials-Prevention. Accessed February 9, 2017.

30. Crawford J, Prado CMM, Johnston MA, et al. Study design and rationale for the phase 3 clinical development program of enobosarm, a selective androgen receptor modulator, for the prevention and treatment of muscle wasting in cancer patients (POWER trials). Curr Oncol Rep 2016;18:37. 